\title{
Celiac Disease in Children: What Has Changed?
}

\author{
Jiri Nevoral* \\ Department of Pediatrics, Charles University, 2nd Medical School and University Hospital Motol, Prague, Czech Republic \\ *Corresponding author: jiri.nevoral@lfmotol.cuni.cz
}

Received January 24, 2014; Revised February 04, 2014; Accepted February 06, 2014

\begin{abstract}
The perception of celiac disease (CD) has changed dramatically during the last two decades, with the introduction of new serological tests with high specificity and sensitivity. CD prevalence in recent years is increasing as it is for other autoimmune diseases. Due to the high prevalence of $C D$, it is one of the most important diseases of the digestive tract. The clinical manifestations of $\mathrm{CD}$ have changed significantly in the last two decades, and these are much more diverse than reported previously. The new European Society for Pediatric Gastroenterology, Hepatology, and Nutrition diagnostic criteria for CD indicate that biopsy can be avoided in strictly selected groups of pediatric patients. The first experiences with these new criteria are positive. A meta-analysis evaluating the relationship between early nutrition and the risk of $\mathrm{CD}$ recommends that both early $(<4$ months) and late $(>7$ months) introduction of gluten to the diet should be avoided, and that gluten should be introduced while the infant is still breastfeeding. The prevalence rates of $\mathrm{CD}$ between two cohorts with different infant feeding practice were significantly different, and the prevalence of $\mathrm{CD}$ in 12-year-old children were significantly reduced, suggesting that prevention of $\mathrm{CD}$ is possible.
\end{abstract}

Keywords: diagnosis, ESPGHAN diagnostic criteria, prevention, screening

Cite This Article: Jiri Nevoral, “Celiac Disease in Children: What Has Changed?” International Journal of Celiac Disease, vol. 2, no. 1 (2014): 18-23. doi: 10.12691/ijcd-2-1-6.

\section{Introduction}

Celiac disease (CD) has been recently defined by the European Society for Paediatric Gastroenterology, Hepatology, and Nutrition (ESPGHAN) as an immunemediated systemic disorder elicited by gluten and related prolamines in genetically susceptible individuals. [1]. It is characterized by the presence of a variable combination of gluten-dependent clinical manifestations, CD-specific antibodies, specific haplotypes of the major histocompatibility complex-human leukocyte antigen (HLA) class II genes (HLA-DQ2 or HLA-DQ8), and enteropathy. CD-specific antibodies comprise autoantibodies against tissue transglutaminase (anti-TG), endomysial antibodies (EMA), and antibodies against deamidated forms of gliadin peptides. [1] CD is the most common, life-long heritable food intolerance worldwide.

The immune response to gluten triggers characteristic lesions in the small intestinal mucosa (atrophy of the villi, crypt hyperplasia, and increased number of intraepithelial lymphocytes [IEL]). The disease occurs in some genetically predisposed individuals who ingest prolamins in their diet, i.e., alcohol soluble proteins rich in glutamine and proline, which are present in wheat, rye, and barley.

The typical presentation of CD is enteropathy, leading to malabsorption of nutrients, which lasts sufficiently long, causing significant malnutrition.

\section{Epidemiology}

Recent new serological tests EMA and anti-TG, with high specificity and sensitivity have dramatically changed the epidemiology and diagnosis of CD. The current prevalence rates of 1:40-1:330 [2] are higher than those of 1:1000-1:3000, which were based on clinical signs and earlier screening data. [3,4] In the first Norwegian study, the nationwide prevalence of clinically diagnosed $C D$ in children at 12 years of age was 5.0:1000 The association between $\mathrm{CD}$ and various comorbidities was found to be 7.1\% (type 1 diabetes mellitus, 4.7\%; Down syndrome, $1.6 \%$; thyroid diseases, $1.4 \%$ ) [5].

Nevertheless, CD remains under-diagnosed in the entire European population. For example, the estimated prevalence of $C D$ in the Czech Republic is 1:200-1:250, i.e., about 40.000-50.000 patients with CD in a population of 10 million inhabitants, but only $15 \%$ are diagnosed to have CD. [6] With the new serological testing, CD is diagnosed at an later age and the clinical presentation has changed. Gastrointestinal manifestations are less often the presenting symptoms. Almost 25\% of children with CD are diagnosed by targeted screening in high risk groups for CD. However, most children with CD remain undiagnosed. [7] Owing to its high prevalence, $C D$ is one of the most important diseases of the digestive tract.

Higher prevalence rates are because of improved diagnostic methods (i.e., the availability of sensitive and specific serological tests), better awareness of CD among physicians and the general population, and greater use of serological tests to identify patients with either extraintestinal or subclinical presentations. [8,9,10] For example, antibody testing for $\mathrm{CD}$ tripled the number of patients diagnosed with $C D$ and quadrupled the median 
age of CD diagnosis. [11] On the other hand, the incidence of classic pediatric CD increased 6.4-fold over 20 years in Scotland, which strongly suggests a real increase in CD incidence [12].

The hygiene hypothesis postulates that the increasing CD prevalence in recent years in Europe and the USA, as well as for other autoimmune diseases, may be owing to reduced bacterial exposure in the first years of life. [13] However, other hypotheses suggest the higher prevalence of CD may be because of infant feeding practices [14,15] and repeated infections. Previous studies have shown an association between the occurrence of CD and infections. $[16,17,18]$ Stene et al. found an increased risk for CD in children with repeated rotavirus infection, as indicated by serum positivity for anti-rotavirus antibodies. [19] However, later studies did not confirm these findings. [20] In a recent population-based, incident case-reference study, an increased CD risk was found in infants who had three or more parent-reported infections before 6 months of age, regardless of the type of infection. [21] A recent Swedish study showed that vaccination at an early age is not a risk factor for CD [22].

\section{Clinical Presentation}

Clinical manifestations of the disease have changed significantly in the last two decades, and these are much more diverse than those reported previously. The symptoms and intensity of symptoms of CD vary widely. It has been estimated that only 1 in every 3 to 1 in every 7 adult CD patient is symptomatic. Most of the cases of CD remain undiagnosed so we have only diagnosed the tip of the iceberg in terms of prevalence. In the same period, a temporal shift in the period at which CD first manifests has been noted, which is often that a later age than that reported previously. Several factors may explain this delay, including prolonged duration of breastfeeding, the later introduction of gluten in the diet, and smaller amount of gluten that children consume. However, other additional, unknown factors may be involved.

Several forms of CD are currently recognized, and their definitions were amended in 2013. [23]:

Pediatric classical CD presents with symptoms and signs of malabsorption, such as diarrhea, steatorrhea, failure to thrive, muscle wasting, poor appetite, abdominal distension, and emotional distress.

Non-classical CD presents without signs and symptoms of malabsorption. Patients with monosymptomatic CD (other than diarrhea or steatorrhea) usually have nonclassical CD. Patients with non-classical CD have bone and muscle manifestations, skin and cutaneous symptoms, neurological and hematological manifestations, disturbances in reproduction, and various other symptoms such as elevated aminotransferase levels.

Subclinical CD (previously silent CD) is below the threshold of clinical detection, i.e. it manifests without sufficient symptoms or signs; therefore, CD testing in routine practice is not initiated.

Asymptomatic CD is not accompanied by symptoms. Individuals with asymptomatic CD are usually found through screening programs in families in which a member was diagnosed with CD or are found on screening patients with disorders associated with a high risk of CD.
Many of these individuals have a reduced quality of life and mild symptoms, which resolve with a gluten-free diet (GFD). These patients do not have asymptomatic CD and should be diagnosed with subclinical CD.

Potential CD is that in which in individuals have a normal, small intestinal mucosa but have positive CD serology. The difficulty in making a CD diagnosis in a patient with potential CD may be compounded by an inadequate biopsy. It is therefore recommended that at least 5 biopsies must be taken from different areas of the mucosa, including one of the duodenal bulb in order to increase the sensitivity in patients whose histological changes are not diffuse. The number of patients with potential $C D$ has recently increased because of the increased awareness of CD and the screening of at-risk groups. Most children with potential CD remain healthy, and in one study, villous atrophy developed in 33\% of children after 3 years of normal diet with gluten [24].

Refractory CD involves persistent or recurrent malabsorptive symptoms and signs with villous atrophy despite the patient being on a strict GFD for more than 12 months. Patients with refractory CD may be negative for EMA and anti-TG. If they are antibody positive, then an undetected gluten exposure should be excluded. Patients with refractory CD are usually adults with typical longterm symptoms. Two types of refractory CD exist: type I with a normal IEL phenotype and type II with clonal expansion of an aberrant IEL population.

\section{Diagnosis}

The diagnostic criteria for CD were first published by the ESPGAN in 1969. [25] A diagnosis of CD was based on three biopsies. In the first biopsy, finding a characteristic histological lesion was necessary to establish a preliminary diagnosis. Then, a second biopsy was performed after the patient had been on a GFD, and resolution of the lesions was required. A third biopsy was performed after gluten challenge, and finding a new histological lesion confirmed the diagnosis. In 1990, the diagnostic criteria were modified such that gluten challenge was limited to children in whom the first biopsy was performed at age less than 2 years, in order to exclude other causes of enteropathy. Gluten challenge was also used when the initial diagnosis was uncertain [26].

In the late 1980s, EMA and anti-TG antibodies were discovered, thus fundamentally changing the understanding of this disease. $[27,28]$ Since then, both immunoglobulin (Ig)A-EMA and IgA-anti-TG were shown to be both highly sensitive and specific for CD (> 95\%). [6,20] Furthermore, correlations between anti-TG levels and histopathologic features of CD have been repeatedly described in children with high titers of anti-TG, directly correlating with villous atrophy. [29,30,31,32] A strong association of CD with HLA-DQ2 and HLA-DQ8 was also found, with a diagnostic sensitivity of $>96 \%$. Consequently, a negative HLA-DQ2 and/or HLA-DQ8 result makes the diagnosis of CD highly unlikely [33].

Based on these studies, the ESPGHAN working group on $\mathrm{CD}$ prepared new guidelines for $\mathrm{CD}$ diagnosis. [1] According to the new ESPGHAN criteria, diagnosis is based on the combined evaluation of symptoms, CDspecific antibodies, histology, and genetics. In patients 
who are symptomatic and have anti-TG titers $>10$ times the upper limit of normal and are positive for EMA, a biopsy is not needed. Additionally, a positive test for HLA-DQ2 and/or HLA-DQ8 is required for the diagnosis of CD. A recent study demonstrated the usefulness of these new criteria. [34] Similarly, we retrospectively found that symptomatic pediatric patients who were positive for EMA and had anti-TG antibodies more than ten times the upper limit of normal, the specificity for Marsh Scores of type 2-3 was 99\%. Results revealed that intestinal biopsies could be omitted in $28 \%$ of patients according to the new ESPGHAN guidelines. Due to the high accuracy of serological tests in combination with clinical symptoms for the diagnosis of CD, this new guideline seems to be applicable even without use of HLA testing. The specificity of serological tests in asymptomatic individuals was much lower (67\%), and it is, therefore, always necessary to perform a biopsy. [35] In a retrospective, multicenter study in Spain, the authors concluded that biopsy could be omitted in 50\% of symptomatic patients without a risk of overdiagnosis (100\% specificity), while in asymptomatic patients, regardless of serology, a biopsy was mandatory [36].

Intestinal biopsy was hitherto the cornerstone for the diagnosis of CD, and avoiding this in selected patients is a revolutionary change. The new ESPGHAN recommendations are therefore not currently accepted without reservations, and some authors have provided critical comments. One is that there are more than 20 different tests to determine anti-TG levels, which can provide substantially varied results, and thus, patients may mistakenly be categorized into a group in which a biopsy is not deemed necessary. [37] One study that discussed the new criteria noted that, in patients in whom the diagnosis of CD is performed without biopsy and in whom endoscopic procedure is avoided, other additional, unexpected diagnoses may be missed in $>10 \%$ of symptomatic patients [38] It was particularly emphasized that symptomatic patients with CD diagnosed without a biopsy must be carefully monitored if their symptoms resolved after instituting a GFD, and if their symptoms do not resolve, then it is necessary to look for other possible diseases [39].

Some authors have criticized the recommendation to begin HLA testing in asymptomatic subjects in an effort to reduce further examinations if HLA-DQ 2/8 is not found. [40] Testing these HLA-DQ alleles is expensive, and the alleles are common in the general population (20-40\%) and CD is present in only $1 \%$. Their routine testing is also not available worldwide. If patients are aware that CD can be diagnosed without the need for biopsy, then some patients with suspected CD may refuse to undergo biopsy It can be also difficult to explaining to parents that a symptomatic child does not require a biopsy while an asymptomatic sibling may require one.

The new recommendations make it obligatory for general practitioners to refer a child with a positive antiTG test to a pediatric gastroenterologist for the final diagnosis of CD. However, there is no guarantee that referral always occurs, and if not, then the number of incorrect diagnoses may increase. The new recommendations provide a new diagnostic opportunity but are still not considered as fixed guidelines and are only help select serologically well-defined symptomatic pediatric patients who do not require biopsy. If a pediatric gastroenterologist does not agree with this diagnostic procedure or all the necessary tests are not available, a biopsy may still be performed to confirm the diagnosis.

\section{Prevention of CD}

Primary prevention focusses on preventing the development of CD. Recently, much attention is paid to the possibility of primary prevention of CD through early nutrition, particularly on the timing and circumstances under which gluten is introduced. [41] A meta-analysis of observational studies concluded that if an infant was breastfed during the introduction of dietary gluten or was breastfed for a prolonged duration, the risk of developing $\mathrm{CD}$ was reduced. Based on previous studies, however, it is not clear whether breast-feeding delays the initiation of CD symptoms or prevents CD. [42] The difference in CD risk between two birth cohorts of comparable age suggests an opportunity for primary prevention. [43] Another metaanalysis evaluating studies that investigated the relationship between early nutrition and the risk of CD recommended that both early ( $<4$ months) and late $(>7$ months) introduction of gluten should be avoided, and that gluten should be introduced while the infant is still breastfed. [44] However, the relationship between breastfeeding infants and the occurrence of $C D$ remains controversial, and only further studies will clarify the relationship [42,45,46].

Nevertheless, the incidence rates of CD between the two birth cohorts differing in infant feeding practices (regarding the age for introduction of gluten-containing complementary foods and the difference in the proportion in infants being introduced to gluten during ongoing breastfeeding) were significantly different, indicating that early CD can be prevented. [47] The significant reduction in prevalence of CD in 12-year-old children suggests that the present infant feeding recommendation to gradually introduce gluten-containing foods from 4 months of age, preferably during ongoing breastfeeding, is favorable [48].

The first, prospective population-based birth cohort with 107,000 children showed that CD had a high risk when gluten introduction was delayed (that is, $>6$ months) as well as when breastfeeding was prolonged (12 months). Patients may develop tolerance by the timely introduction of gluten, but elucidating the factors involved in the loss of tolerance needs future studies [49].

Secondary prevention targets early diagnosis of the disease with the option of preventing disease progression or reducing the severity of symptoms. However, mass screening for $\mathrm{CD}$ in the general population is not recommended primarily because the natural course of CD is not well known. Currently, it is not clear whether cases found by screening have the same risk for long-term complications as clinically diagnosed patients. Similarly, it is unclear whether asymptomatic patients should be treated, and whether they may find it difficult to adhere to GFD. In contrast, the higher incidence of other forms of CD than that of classical forms, significant risks associated with late diagnosis and therapy in CD associated with autoimmune diseases, and severe, CDrelated complications require the introduction of targeted screening for CD. Therefore, testing certain groups of patients with high risk of $\mathrm{CD}$ has been advocated, 
particularly in patients at risk of disease and risk groups, such as those with probable symptoms, autoimmune diseases, and other possible complications. Screening is also recommended among first-degree relatives of individuals with CD, even if they are asymptomatic.

Mass screening is not recommended owing to the above-mentioned reasons and because case finding in high-risk groups does not detect all patients with CD, and a large proportion of patients remain undiagnosed; therefore, the methods for improving this situation are required. One solution could be to determine the levels of anti-TG with parental consent among children whose blood samples are occasionally taken for other reasons [50].

The early diagnosis of CD can also be achieved by promoting greater awareness among health professionals and the public about the disease by using methodological protocols in the form of recommendations for all physicians and through the early use of serological tests in symptomatic patients. The effectiveness of educational campaigns was demonstrated in a study in Finland, with an estimated CD detection of 70\% [51].

Tertiary prevention aims to reduce the negative impact of already existing diseases. Since GFD reduces complications related to this disease, it may be considered tertiary prevention.

\section{Treatment}

Strict lifelong GFD involving the complete elimination of wheat, rye, and barley is the only casual therapy for CD. The clinical response to a GFD in symptomatic patients is often dramatic and occurs within few weeks. Mucosa in children usually returns to normal after about 6 months. However, GFD is a strict regime, which requires lifelong exclusion of raw materials, foods, and beverages containing gluten. Maintaining a GFD requires a significant and permanent change in lifestyle. In this situation, any extension in the assortment of raw materials by alternative cereal is desirable. For this, oats, which increases a diet's nutritional value, has been considered, but their use remains controversial. Contamination of oats during production and processing with prolamins of other cereals is common and it is essential that patients use only uncontaminated oats. Some clinical and experimental studies show that $5 \%$ of CD patients may not even tolerate pure oats, which are labeled gluten free. We recommend starting treatment with a strict GFD diet, excluding oats. After symptoms disappear and no antibodies specific for $\mathrm{CD}$ are detected in the serum, gluten-free oats can be introduced into the GFD under careful clinical and laboratory monitoring. [52] We suggest the need to select oat cultivars with low avenin content and to develop recombinant oat varieties complying with these requirements. Assays for detecting avenins in oat products are also needed, and guidelines for the processing of oats and manufacture of oat products, as well as recommendations for follow-up of patients with CD who consume oats are needed [53].

Future prospects for the treatment of CD may include the production of genetically modified grains without biologically active peptide sequences, the development of drugs that block tissue transglutaminase or immunomodulate antigenic polypeptides, or some other method.

\section{Conclusion}

CD is a common treatable illness with many different symptoms and serious complications, with an increasing prevalence. Despite this, CD remains significantly underdiagnosed in the general population, especially among adults. New diagnostic criteria offer the potential to omit a diagnostic biopsy in selected symptomatic pediatric patients. Significant differences in the prevalence of CD between the two cohorts differing in infant feeding practice suggest an opportunity for primary prevention. Targeted serological screening programs aimed at patients at risk and those with diseases, suspicious symptoms, or associated autoimmune diseases is the most important measure required to improve the present situation, in which CD is underdiagnosed. .Case finding in groups of patients with high risk for CD is advocated.

\section{Acknowledgements}

Supported by a research grant from the University Hospital Motol (VZ FNM) 64203/6001.

The author reports that there are no conflicts of interests to declare.

\section{References}

[1] Husby, S., Koletzko, S., Korponay-Szabó, I.R., Mearin, M.L., Phillips, A., Shamir, R., Troncone, R., Giersiepen, K., Branski, D., Catassi, C., Lelgeman, M., Mäki, M., Ribes-Koninckx, C., Ventura, A., Zimmer, K.P., "European Society for Pediatric Gastroenterology, Hepatology, and Nutrition guidelines for the diagnosis of coeliac disease," J Pediatric Gastroenterol Nutr, 54. 136-160. 2012.

[2] Mustalahti, K., Catassi, C., Reunanen, A., Fabiani, E., Heier, M., McMillan, S., Murray, L., Metzger, M.H., Gasparin, M., Bravi, E., Mäki, M., „The prevalence of celiac disease in Europe: results of a centralized, international mass screening project," Ann. Med, 42 (8). 587-595. 2010.

[3] Lohi, B., Mustalahti, K., Kaukinen, K., Laurila, K., Collin, P., Rissanen, H., Lohi, O., Bravi, E., Gasparin, M., Reunanen, A., Mäki, M., „Increasing prevalence of coeliac disease over time,” Aliment Pharmacol Ther, 26 (9). 1217-1225. 2007.

[4] Rubio-Tapia, A., Kyle, R.A,, Kaplan, E.L., Johnson, D.R., Page, W., Erdtmann, F., Brantner, T.L., Kim, W.R., Phelps, T.K., Lahr, B.D., Zinsmeister, A.R., Melton, L.J. 3rd, Murray, J.A., "Increased prevalence and mortality in undiagnosed celiac disease,” Gastroenterology, 137 (1). 88-93. 2009.

[5] Størdal, K., Bakken, I.J, Surén, P., Stene, L.C., "Epidemiology of coeliac disease and comorbidity in Norwegian children,” J Pediatr Gastroenterol Nutr, 57(4). 467-71. Oct.2013.

[6] Frič, P., Nevoral, J., “Cílený screening celiakie,” Interní Med, 11(11). 484-487. 2009.

[7] Ravikumara, M., Tuthill, D.P., Jenkins, H.R., "The changing clinical presentation of coeliac disease,” Arch Dis Child, 91(12). 969-71. Dec.2006.

[8] Steens, R.F., Csizmadia, C.G., George, E.K., Ninaber, M.K., Hira Sing, R.A., Mearin, M.L., "A national prospective study on childhood celiac disease in the Netherlands 1993-2000: an increasing recognition and a changing clinical picture," J Pediatr, 147(2). 239-43. Aug.2005.

[9] Roma, E., Panayiotou, J., Karantana, H., Constantinidou, C., Siakavellas, S.I., Krini, M., Syriopoulou, V.P., Bamias, G., "Changing pattern in the clinical presentation of pediatric celiac disease: a 30-year study,” Digestion, 80(3). 185-91. 2009.

[10] Telega, G., Bennet, T.R., Werlin, S. "Emerging new clinical patterns in the presentation of celiac disease," Arch Pediatr Adolesc Med, 162(2).164-8. Feb.2008.

[11] McGowan, K.E., Castiglione, D.A., Butzner, J.D. “The changing face of childhood celiac disease in North America: Impact of serological testing,” Pediatrics, 124(6). 1572-8. Dec. 2009. 
[12] White, L.E., Merrick, V.M., Bannerman, E., Russell, R.K., Basude, D., Henderson, P., Wilson, D.C., Gillett, P.M., "The rising incidence of celiac disease in Scotland," Pediatrics, 132(4). e92431. Oct.2013.

[13] Bach, J.F., "The effect of infections on susceptibility to autoimmune and allergic diseases,” N Engl J Med, 347(12). 91120. Sep.2002.

[14] Ivarsson, A., Myléus, A., Norström, F., van der Pals, M., Rosén, A., Högberg, L., Danielsson, L., Halvarsson, B., Hammarroth, S., Hernell, O., Karlsson, E., Stenhammar, L., Webb, C.H., Sandström, O., Carlsson, A., "Prevalence of Childhood Celiac Disease and Changes in Infant Feeding," Pediatrics, 131. e687e694. 2013.

[15] Størdal, K., White, R.A., Eggesbø, M., “Early Feeding and Risk of Celiac Disease in a Prospective Birth Cohort," Pediatrics, 132. e1202-1209. 2013.

[16] Sandberg-Bennich, S., Dahlquist, G., Kallen, B., "Coeliac disease is associated with intrauterine growth and neonatal infections," Acta Paediatr; 91(1). 30-33. 2002.

[17] Wingren, C.J., Bjorck, S., Lynch, K.F., Ohlsson, H., Agardh, D., Merlo, J., "Coeliac disease in children: a social epidemiological study in Sweden,” Acta Paediatr, 101(2). 185-191. 2011.

[18] Olen, O., Bihagen, E., Rasmussen, F., Ludvigsson, J.F., "Socioeconomic position and education in patients with coeliac disease,” Dig Liver Dis, 44(6). 471-476. 2012.

[19] Stene, L.C., Honeyman, M.C., Hoffenberg, E.J., Haas, J.E., Sokol, R.J., Emery, L., Taki, I., Norris, J.M., Erlich, H.A., Eisenbarth, G.S., Rewers, M., "Rotavirus infection frequency and risk of celiac disease autoimmunity in early childhood: a longitudinal study,” Am J Gastroenterol, 101(10). 2333-2340. 2006.

[20] Welander, A., Tjernberg, A.R., Montgomery, S.M., Ludvigsson, J., Ludvigsson, J.F., "Infectious disease and risk of later celiac disease in childhood," Pediatrics, 125(3). e530-536. 2010.

[21] Myléus, A., Hernell, O., Gothefors, L., Hammarström, M.L., Persson, L.Å., Stenlund, H., Ivarsson, A., "Early infections are associated with increased risk for celiac disease: an incident casereferent study,” BMC Pediat, 12. 194 Dec.2012.

[22] Myléus, A., Stenlund, H., Hernell, O., Gothefors, L., Hammarström, M.L., Persson, L.A., Ivarsson, A., "Early Vaccinations Are Not Risk Factors for Celiac Disease,” Pediatrics 130. e63. 2012.

[23] Ludvigsson, J.F., Leffler, D.A., Bai, J.C., Biagi, F., Fasano, A., Green, P.H., Hadjivassiliou, M., Kaukinen, K., Kelly, C.P., Leonard, J.N., Lundin, K.E., Murray, J.A, Sanders, D.S, Walker, M.M., Zingone, F., Ciacci, C., "The Oslo definitions for coeliac disease and related terms,” Gut, 62(1). 43-52. Jan.2013.

[24] Tosco A, Salvati VM, Auricchio R, Maglio, M., Borrelli, M., Coruzzo, A., Paparo, F., Boffardi, M., Esposito, A., D'Adamo, G., Malamisura, B., Greco, L., Troncone, R., "Natural History of Potential Celiac Disease in Children,” Clin Gastroenterol Hepatol, 9. 320-325. 2011.

[25] Meeuwisse, G.W., "Diagnostic criteria in coeliac disease,” Acta Paediatr Scand, 59. 461-3. 1970.

[26] Walker-Smith, J., Guandalini, S., Schmitz, J., Shmerling, D., Visakorpi, J. "Revised criteria for diagnosis of coeliac disease. Report of Working Group of European Society of Paediatric Gastroenterology and Nutrition,” Arch Dis Child, 65. 909-911. 1990.

[27] Grodzinsky, E., Jansson, G., Skogh, T., Stenhammar, L., FalthMagnusson, K., "Anti-endomysium and anti-gliadin antibodies as serological markers for coeliac disease in childhood: a clinical study to develop a practical routine,” Acta Paediatr, 84. 294-8. 1995.

[28] Dieterich, W., Ehnis, T., Bauer, M., Donner, P., Volta, U., Riecken, E.O., Schuppan, D., „Identification of tissue transglutaminase as the autoantigen of celiac disease," Nat Med, 3. 797-801. 1997.

[29] Barker, C.C., Mitton, C., Jevon, G., Mock, T., “Can tissue transglutaminase antibody titers replace small-bowel biopsy to diagnose celiac disease in select pediatric populations?” Pediatrics 115. 1341-6. 2005.

[30] Dahlbom, I., Korponay-Szabó, I.R., Kovacs, J.B., Szalai, Z., Mäki, M., Hansson T., "Prediction of clinical and mucosal severity of coeliac disease and dermatitis herpetiformis by quantification of IgA/IgG serum antibodies to tissue transglutaminase," J Pediatr Gastroenterol Nutr, 50.140-6. 2010.

[31] Donaldson, M.R., Firth, S.D., Wimpee, H., Leiferman, K.M., Zone, J.J., Horsley, W., O'Gorman, M.A., Jackson, W.D., Neuhausen,
S.L., Hull, C.M., Book, L.S., “Correlation of duodenal histology with tissue transglutaminase and endomysial antibody levels in pediatric celiac disease," Clin Gastroenterol Hepatol, 5. 567-73. 2007.

[32] Donaldson, M.R., Book, L.S., Leiferman, K.M., Zone, J.J, Neuhausen, S.L., "Strongly positive tissue transglutaminase antibodies are associated with Marsh 3 histopathology in adult and pediatric celiac disease," J Clin Gastroenterol, 42. 256-60-73. 2008.

[33] Margaritte-Jeannin, P., Babron, M.C., Bourgey, M., Louka, A.S., Clot, F., Percopo, S., Coto. I., Hugot, J.P., Ascher, H., Sollid, L.M., Greco, L., Clerget-Darpoux, F., "HLA-DQ relative risks for coeliac disease in European populations: a study of the European Genetics Cluster on Coeliac Disease,” Tissue Antigens, 63. 562-7. 2004.

[34] Klapp, G., Masip, E., Bolonio, M., Donat, E., Polo, B., Ramos, D., Ribes-Koninckx, C., "Celiac disease: the new proposed ESPGHAN diagnostic criteria do work well in a selected population,” J Pediatr Gastroenterol Nutr, 56. 251-256. 2013.

[35] Nevoral, J., Kotalova, R., Hradsky, O., Valtrova, V., Zarubova, K., Lastovicka, J., Neubertova, E., Trnkova, M., Bronsky, J., "Symptom positivity is essential for omitting biopsy in children with suspected celiac disease according to the new ESPGHAN guidelines,” Eur J Pediatr. Nov. 2013 [Epub ahead of print] PMID: 24233405.

[36] Donat, E., Ramos, J.M., Sanchez-Valverde, F., Moreno, A., Martinez, M., Leis, R., Pena, L., Castillejo, G., Fernandez, S., Garcia, Z., Ortigosa, L., Balmaseda, E., Marguán, J.M., Eizaguirre, J., Loroenzo, H., Barrio, J., Ribes-Koninckx, C., "ESPGHAN 2012 Coeliac disease diagnostic criteria validation: a retrospective spanish multicentric study," in the 21st United European Gastroenterology Week, Berlin 2013.

[37] Egner, W., Shrimpton, A., Sargur, R., Patel, D., Swallow, K., "ESPGHAN guidance on coeliac disease 2012: multiples of ULN for decision making do not harmonise assay performance across centers,” J Pediatr Gastroenterol Nutr, 55(6). 733-5. Dec.2012.

[38] Guandalini, S., Newland, C., "Can We Really Skip the Biopsy in Diagnosing Symptomatic Children With Celiac Disease,” J Pediatr Gastroenterol Nutr, 57(4). e24. 2013.

[39] Husby, S., Koletzko, S, Korponay-Szabó, I., “Authors’ Response. Letters to the Editor,” J Pediatr Gastroenterol Nutr, 57(4). e24. 2013.

[40] Hill, I.D., "Screening for celiac disease," J Pediatr Gastroenterol Nutr, 57(4). 414-415. 2013.

[41] Troncone, R., Ivarsson, A., Szajewska, H., Mearin, M.L. and the Members of European Multistakeholder Platform on CD (CDEUSSA), "Review article: future research on coeliac disease a position report from the European multistakeholder platform on coeliac disease (CDEUSSA)," Aliment Pharmacol Ther, 27. 103043. 2008.

[42] Akobeng, A.K., Ramanan, A.V., Buchan, I., Heller, R.F.. “Effect of breast feeding on risk of coeliac disease: a systematic review and metaanalysis of observational studies," Arch Dis Child, 91(1). 39-43. 2006.

[43] Olsson, C., Stenlund, H., Hörnell. A., Hernell, O., Ivarsson, A., "Regional variation in celiac disease risk within Sweden revealed by the nationwide prospective incidence register," Acta Paediatr, 98(2). 337-342. 2009.

[44] Szajewska H, Chmielewska A, Piescik-Lech M, Ivarsson A, Kolacek S, Koletzko S, Mearin ML, Shamir R, Auricchio R, Troncone R., PREVENTCD Study Group. "Systematic review: early infant feeding and the prevention of coeliac disease," Aliment Pharmacol Ther, 36(7). 607-618. 2012.

[45] Silano, M., Agostoni, C., Guandalini, S., "Effect of the timing of gluten introduction on the development of celiac disease," World J Gastroenterol, 16(16). 1939-1942. 2010.

[46] Ludvigsson, J.F, Fasano, A., “Timing of introduction of gluten and celiac disease risk,” Ann Nutr Metab, 60(suppl 2). 22-29. 2012.

[47] Olsson, C., Hernell, O., Hörnell, A., Lönnberg, G., Ivarsson, A., "Difference in celiac disease risk between Swedish birth cohorts suggests an opportunity for primary prevention,” Pediatrics, 122. 528-534. 2008.

[48] Ivarsson, A., Myléus, A., Norström, F., van der Pals, M., Rosén, A., Högberg L., Danielsson, L., Halvarsson, B., Hammarroth, S., Hernell, O., Karlsson, E., Stenhammar, L., Webb, C.H., Sandström, O., Carlsson, A., "Prevalence of Childhood Celiac Disease and Changes in Infant Feeding,” Pediatrics, 131. e687e694. 2013. 
[49] Størdal, K., White, R.A., Eggesbø, M., "Early Feeding and Risk of Celiac Disease in a Prospective Birth Cohort," Pediatrics, 132. e 1202-1209. 2013.

[50] Catassi, C., Lionetti, E., „Case finding for celiac disease is okay, but is it enough?” J Pediatr Gastroenterol Nutr, 57(4). 415-7. Oct. 2013.

[51] Collin, P., Huhtala, H., Virta, L., Kekkonen, L., Reunala, T., "Diagnosis of celiac disease in clinical practice: physician's alertness to the condition essential," J Clin Gastroenterol, 41(2). 152-6. Feb. 2007.

[52] Murch, S., Jenkins, H., Auth, M. Bremner, R., Butt, A., France, S., Furman, M., Gillett, P., Kiparissi, F., Lawson, M., McLain, B., Morris, M.A., Sleet, S., Thorpe, M., "Joint BSPGHAN and Coeliac UK guidelines for the diagnosis and management of coeliac disease in children," Arch Dis Child, 98(10). 806-11. Oct. 2013.

[53] Fric, P., Gabrovska, D., Nevoral, J., "Celiac disease, gluten-free diet, and oats,” Nutr Rev. 69(2). 107-15. Feb.2011. 